\title{
Cytotoxicity of some synthetic bis(arylidene) derivatives of cyclic ketones towards cisplatin-resistant human ovarian carcinoma cells
}

\author{
Hinal Patel ${ }^{1} \cdot$ Begum Mothia $^{1} \cdot$ Jaison Patel ${ }^{1} \cdot$ Olatunde Fasanya $^{1} \cdot$ Kartheek Sooda $^{2} \cdot$ Farideh Javid $^{2} \cdot$ Peter B. Wyatt $^{1}$
}

Received: 16 July 2019 / Accepted: 13 March 2020 / Published online: 17 April 2020

(c) The Author(s) 2020

\begin{abstract}
Symmetrical $\alpha, \alpha^{\prime}$-bis(arylidene)ketones were prepared by acid-catalyzed aldol condensations between aliphatic ketones (e.g., cyclopentanone, 4-alkylcyclohexanones, tetrahydropyran-4-one, and tetrahydrothiopyran-4-one) and two equivalents of an aromatic hydroxyaldehyde (e.g., 4-hydroxybenzaldehyde, 3,4-dihydroxybenzaldehyde, vanillin, isovanillin, and 3-fluoro-4hydroxybenzaldehyde). Most of the compounds were cytotoxic towards the cisplatin-resistant human ovarian cancer cell line A2780-CP70 as well as the non-resistant line A2780.
\end{abstract}

Keywords Ovarian cancer $\cdot$ Dienone $\cdot$ Cytotoxicity $\cdot$ Curcumin $\cdot$ Carboplatin $\cdot$ Cisplatin

\section{Introduction}

Ovarian cancer is estimated to kill more than 180,000 women each year worldwide (World Health Organization 2019). A particular challenge is the frequent development of resistance towards cisplatin, one of the important chemotherapeutic agents (Galluzzi et al. 2014). Curcumin (1), which is a major constituent of the spice turmeric, inhibits the proliferation of cisplatin-resistant ovarian cancer cells by multiple mechanisms (Weir et al. 2007). Although curcumin has low toxicity, its low potency, rapid metabolism, and low oral availability limit its clinical use and have prompted the evaluation of analogues, particularly $\alpha, \alpha^{\prime}$-bis (arylidene)ketones (Adams et al. 2004, Selvendiran et al. 2007). Such compounds are easily prepared through chemical synthesis (Scheme 1) and considerable structural diversity can be obtained by combining sets of readily available starting materials. In our present study we have

Farideh Javid

fajavid@hud.ac.uk

$\triangle$ Peter B. Wyatt

p.b.wyatt@qmul.ac.uk

1 Department of Chemistry, School of Biological and Chemical Sciences, Queen Mary University of London, Joseph Priestley Building, Mile End Road, London E1 4NS, UK

2 Department of Pharmacy, School of Applied Sciences, University of Huddersfield, Huddersfield HD1 3DH, UK prepared a group of symmetrically substituted bis(arylidene)ketones containing new examples along with some previously known compounds. We have then examined the ability of these substances to inhibit the growth of both A2780 human ovarian carcinoma cells and the related cisplatin-resistant cells, A2780-CP70.

\section{Material and methods}

'Petrol' refers to the fraction of petroleum spirit with bp $40-60{ }^{\circ} \mathrm{C}$. Flash chromatography was performed on BDH silica gel $(33-70 \mu \mathrm{m})$. All new compounds were $>95 \%$ pure as assessed by TLC and high field NMR. Melting points were determined using a Reichert hot stage microscope and are uncorrected. NMR spectra were recorded on Jeol EX270 and Bruker AMX400 spectrometers. Mass spectra were provided by the EPSRC NMSF at Swansea University. Low resolution, EI and CI measurements were performed on a Quattro II triple quadrupole instrument. High resolution measurements were made using a Finnigan MAT 900 XCT high resolution spectrometer. DEPT and/or HSQC experiments were used to assist the assignment of all reported ${ }^{13} \mathrm{C}$ NMR spectra.

Aldol condensations to prepare the bis(arylidene)ketones were performed by analogy with existing literature (Sardjiman et al. 1997, Youssef et al. 2004) using the conditions and product isolation procedures exemplified for the preparation of ketone $\mathbf{1 0}$ below. The syntheses of the following aldol products have been reported previously: $\mathbf{2}$ (Leow et al. 
Scheme 1 General scheme for formation of bis(arylidene) ketone analogs of curcumin (1) by aldol condensation<smiles>COc1cc(/C=C/C(=O)CC(=O)/C=C/c2ccc(O)c(OC)c2)ccc1O</smiles>

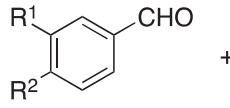

Substituted benzaldehydes
2014), 3 (Sardjiman et al. 1997), 5 (Leow et al. 2014), 6 (Sardjiman et al. 1997), 8 (Inayama et al. 1976), 14 (Bairam et al. 2017), 16 (Costi et al. 2004, Artico et al. 1998), 19 (Tan et al. 2014).

\section{2,5-bis(3-fluoro-4-hydroxybenzylidene) cyclopentanone (4)}

3-Fluoro-4-hydroxybenzaldehyde $(0.90 \mathrm{~g}, 6.42 \mathrm{mmol})$ and cyclopentanone $(0.29 \mathrm{ml}, 3.21 \mathrm{mmol})$ gave the title compound 4 as a green powder $(0.19 \mathrm{~g}, 18 \%)$; mp $289^{\circ} \mathrm{C}$ (decomp, from EtOH); $v_{\max }(\mathrm{KBr}) / \mathrm{cm}^{-1} 3373(\mathrm{O}-\mathrm{H}), 1653$ $(\mathrm{C}=\mathrm{O}), 1578(\mathrm{C}=\mathrm{C}) ; \delta_{\mathrm{H}}\left(400 \mathrm{MHz}, \mathrm{DMSO}-d_{6}\right), 3.09(4 \mathrm{H}$, $\mathrm{s}, \mathrm{H}-3,4), 7.12\left(2 \mathrm{H}, \mathrm{t}, J 9 \mathrm{~Hz}, \mathrm{H}-5^{\prime}, 5^{\prime \prime}\right), 7.40(2 \mathrm{H}, \mathrm{s}, \mathrm{CH}=)$, 7.44 (2H, d, J 9 Hz, H-6', 6), 7.55 (2H, d, J $\left.13 \mathrm{~Hz}, \mathrm{H}-2^{\prime}, 2^{\prime \prime}\right)$, $10.6\left(2 \mathrm{H}\right.$, br s, OH); $\delta_{\mathrm{C}}\left(101 \mathrm{MHz}, \mathrm{DMSO}-d_{6}\right) 25.7(\mathrm{C}-3,4)$, 118.0 (d, $J 3 \mathrm{~Hz}, \mathrm{CH}), 118.1$ (d, J $12 \mathrm{~Hz}, \mathrm{CH}), 127.2$ (d, $J$ $6 \mathrm{~Hz}, \mathrm{C}_{\text {quat }}$ ), 128.0 (d, J $\left.3 \mathrm{~Hz}, \mathrm{CH}\right), 131.5$ (d, J $2 \mathrm{~Hz}, \mathrm{CH}$ ), 135.8 (= $\mathrm{C}_{\text {quat }}$ ), 146.5 (d, J $\left.13 \mathrm{~Hz}, \mathrm{C}-\mathrm{O}\right), 150.8$ (d, J $242 \mathrm{~Hz}$, $\mathrm{CF}), 194.8(\mathrm{C}=\mathrm{O}) ; \delta_{\mathrm{F}}\left(376 \mathrm{MHz}, \mathrm{DMSO}-d_{6}\right),-132.0$; HRMS (ESI) $\mathrm{m} / \mathrm{z}$ found 329.0990; calculated for $\mathrm{C}_{19} \mathrm{H}_{15} \mathrm{~F}_{2} \mathrm{O}_{3}\left(\mathrm{M}+\mathrm{H}^{+}\right) 329.0984$.

\section{2,6-bis(3-fluoro-4-hydroxybenzylidene) cyclohexanone (7)}

3-Fluoro-4-hydroxybenzaldehyde $(0.90 \mathrm{~g}, 6.42 \mathrm{mmol})$ and cyclohexanone $(0.33 \mathrm{~mL}, 3.21 \mathrm{mmol})$, and methanol $(3 \mathrm{~mL})$ gave the title compound 7 as a green powder $(0.05 \mathrm{~g}, 5 \%), \mathrm{mp}$ 236-240 ${ }^{\circ} \mathrm{C}$ (from EtOH); $v_{\max }(\mathrm{KBr}) / \mathrm{cm}^{-1} 3366(\mathrm{O}-\mathrm{H}), 1653$ $(\mathrm{C}=\mathrm{O}), 1590(\mathrm{C}=\mathrm{C}) ; \delta_{\mathrm{H}}\left(400 \mathrm{MHz}, \mathrm{DMSO}-d_{6}\right), 1.73(2 \mathrm{H}$, quintet, $J 6 \mathrm{~Hz}, \mathrm{H}-4), 2.86(4 \mathrm{H}, \mathrm{t}, J 6 \mathrm{~Hz}, \mathrm{H}-3,5), 7.03(2 \mathrm{H}, \mathrm{t}, J$ $\left.9 \mathrm{~Hz}, \mathrm{H}-5^{\prime}, 5^{\prime \prime}\right), 7.24$ (2H, d, $\left.J 8 \mathrm{~Hz}, 6^{\prime}, 6^{\prime \prime}\right), 7.37$ (2H, dd, $J 13$, $\left.2 \mathrm{~Hz}, \mathrm{H}-2^{\prime}, 2^{\prime \prime}\right), 7.52(2 \mathrm{H}, \mathrm{s}, \mathrm{CH}=), 10.4(2 \mathrm{H}$, br s, OH $) ; \delta_{\mathrm{C}}$ (101 MHz, DMSO-d $) 22.3$ (C-4), 27.7 (C-3, 5), 117.7 (d, J $3 \mathrm{~Hz}, \mathrm{CH}), 118.0$ (d, J $18 \mathrm{~Hz}, \mathrm{CH}), 127.0\left(\mathrm{~d}, J 6 \mathrm{~Hz}, \mathrm{C}_{\text {quat }}\right)$ 127.7 (d, $3 \mathrm{~Hz}, \mathrm{CH}), 134.5$ (= $\left.\mathrm{C}_{\text {quat }}\right), 134.8(\mathrm{CH}=), 145.9$ (d, $J$ $12 \mathrm{~Hz}, \mathrm{C}-\mathrm{O}), 150.6$ (d, J $241 \mathrm{~Hz}, \mathrm{CF}), 188.4(\mathrm{C}=\mathrm{O}) . \delta_{\mathrm{F}}$ $\left(376 \mathrm{MHz}\right.$, DMSO- $\left.d_{6}\right)$-132.3; HRMS (ESI) $\mathrm{m} / \mathrm{z}$ found 343.1141; calculated for $\mathrm{C}_{20} \mathrm{H}_{17} \mathrm{~F}_{2} \mathrm{O}_{3}\left(\mathrm{M}+\mathrm{H}^{+}\right) 343.1140$.

\section{2,6-bis(3-fluoro-4-hydroxybenzylidene)-4- methylcyclohexanone (9)}

3-Fluoro-4-hydroxybenzaldehyde $(0.9 \mathrm{~g}, 6.42 \mathrm{mmol})$ and 4-methylcyclohexanone $(0.39 \mathrm{ml}, 3.21 \mathrm{mmol})$ gave title compound 9 as a yellow powder $(0.36 \mathrm{~g}, 31 \%)$; $\mathrm{mp}$ $163-165^{\circ} \mathrm{C}$ (from EtOH); $v_{\max }(\mathrm{KBr}) / \mathrm{cm}^{-1} 3134(\mathrm{O}-\mathrm{H})$, $1652(\mathrm{C}=\mathrm{O}), 1589(\mathrm{C}=\mathrm{C}) ; \delta_{\mathrm{H}}\left(400 \mathrm{MHz}, \mathrm{DMSO}-d_{6}\right) 1.05$ $\left(3 \mathrm{H}, \mathrm{d}, J 7 \mathrm{~Hz}, \mathrm{CH}_{3}\right), 1.78-1.82$ (1H, m, H-4), 2.50-2.53 $\left(2 \mathrm{H}, \mathrm{m}\right.$, overlapping with DMSO- $\left.d_{5}, \mathrm{H}-3_{\mathrm{ax}}, 5_{\mathrm{ax}}\right), 2.94(2 \mathrm{H}$, dd, $\left.J 16,3 \mathrm{~Hz}, \mathrm{H}-3_{\text {eq }}, 5_{\text {eq }}\right), 7.03\left(2 \mathrm{H}, \mathrm{t}, J 9 \mathrm{~Hz}, \mathrm{H}-5^{\prime}, 5^{\prime \prime}\right)$, $7.23\left(2 \mathrm{H}, \mathrm{dd}, J 2,9 \mathrm{~Hz}, 6^{\prime}, 6^{\prime \prime}\right), 7.37(2 \mathrm{H}, \mathrm{dd}, J 13,2 \mathrm{~Hz}, \mathrm{H}-$ $\left.2^{\prime}, 2^{\prime \prime}\right), 7.51(2 \mathrm{H}, \mathrm{s}, \mathrm{CH}=), 10.4(2 \mathrm{H}$, br s, OH $) ; \delta_{\mathrm{C}}$ (101 MHz, DMSO- $\left.d_{6}\right), 21.3(\mathrm{Me}), 28.6$ (C-4), 35.6 (C-3, 5), 117.7 (d, J $3 \mathrm{~Hz}, \mathrm{CH}), 118.0$ (d, J $18 \mathrm{~Hz}, \mathrm{CH}), 127.0$ (d, J $\left.6 \mathrm{~Hz}, \mathrm{C}_{\text {quat }}\right), 127.7$ (d, J $\left.3 \mathrm{~Hz}, \mathrm{CH}\right), 133.6\left(\mathrm{C}_{\text {quat }}\right), 135.1$ (d, J $2 \mathrm{~Hz},=\mathrm{CH}), 145.9$ (d, J $12 \mathrm{~Hz}, \mathrm{C}-\mathrm{O}), 150.6(\mathrm{~d}, J 241 \mathrm{~Hz}$, $\mathrm{CF}), 188.2(\mathrm{C}=\mathrm{O}) ; \delta_{\mathrm{F}}\left(376 \mathrm{MHz}, \mathrm{DMSO}-d_{6}\right)-132.3$; HRMS (ESI) $m / z$ found 357.1294; calculated for $\mathrm{C}_{21} \mathrm{H}_{19} \mathrm{~F}_{2} \mathrm{O}_{3}\left(\mathrm{M}+\mathrm{H}^{+}\right) 357.1297$.

\section{2,6-bis(4-hydroxybenzylidene)-4- ethylcyclohexanone (10)}

4-Hydroxybenzaldehyde $(1.22 \mathrm{~g}, 10 \mathrm{mmol})$ and 4ethylcyclohexanone $(0.63 \mathrm{~g}, 5 \mathrm{mmol})$ were stirred together in methanol $(5 \mathrm{~mL})$ at $30^{\circ} \mathrm{C}$ until a clear solution was obtained. Concentrated hydrochloric acid $(1 \mathrm{~mL})$ was added and the mixture was stirred at $30{ }^{\circ} \mathrm{C}$ for 1 day. The mixture was then treated with cold $\mathrm{AcOH}$-water (20 mL; 1:1), and filtered. The precipitate was purified by recrystallization from methanol in the presence of charcoal to give 2,6-bis(4-hydroxybenzylidene)-4-ethylcyclohexanone (10) (0.83 g, 50\%), as yellow crystals; $\mathrm{mp}$ $229-231^{\circ} \mathrm{C}$ (from MeOH); $v_{\max }(\mathrm{Nujol}) / \mathrm{cm}^{-1} 3363$ $(\mathrm{OH}), 1627(\mathrm{C}=\mathrm{O}), 1573(\mathrm{C}=\mathrm{C}) ; \delta_{\mathrm{H}}(400 \mathrm{MHz}, \mathrm{DMSO}-$ $\left.d_{6}\right), 0.85\left(3 \mathrm{H}, \mathrm{t}, J 7 \mathrm{~Hz}, \mathrm{CH}_{3} \mathrm{CH}_{2}\right), 1.37$ (2H, quintet, $J$ $\left.7 \mathrm{~Hz}, \mathrm{CH}_{2} \mathrm{CH}_{3}\right), 1.51-1.59(1 \mathrm{H}, \mathrm{m}, \mathrm{H}-4), 2.51(2 \mathrm{H}, \mathrm{t}, J$ $\left.13 \mathrm{~Hz}, \mathrm{H}-3_{\mathrm{ax}}, 5_{\mathrm{ax}}\right), 3.00\left(2 \mathrm{H}, \mathrm{d}, J 13 \mathrm{~Hz}, \mathrm{H}-3_{\mathrm{eq}}, 5_{\mathrm{eq}}\right), 6.87$ $\left(4 \mathrm{H}, \mathrm{d}, J 9 \mathrm{~Hz}, \mathrm{H}-3^{\prime}, 5^{\prime}, 3^{\prime \prime}, 5^{\prime \prime}\right), 7.41(4 \mathrm{H}, \mathrm{d}, J 9 \mathrm{~Hz}, \mathrm{H}-$ 
$\left.2^{\prime}, 6^{\prime}, 2^{\prime \prime}, 6^{\prime \prime}\right), 7.57(2 \mathrm{H}, \mathrm{s},=\mathrm{CH}), 9.94(2 \mathrm{H}, \mathrm{s}, \mathrm{OH}) ; \delta_{\mathrm{C}}$ $\left(101 \mathrm{MHz}, \mathrm{DMSO}-d_{6}\right), 11.3\left(\mathrm{CH}_{3} \mathrm{CH}_{2}\right), 27.8\left(\mathrm{CH}_{3} \mathrm{CH}_{2}\right)$, 33.4 (C-3, 5), 34.9 (C-4), $115.5(\mathrm{CH}), 126.4\left(\mathrm{C}_{\text {quat }}\right)$, $132.31(\mathrm{CH}), 132.35(\mathrm{CH}), 136.1(\mathrm{CH}), 158.2(\mathrm{C}-\mathrm{O})$, $188.4(\mathrm{C}=\mathrm{O})$; HRMS (ESI) $\mathrm{m} / \mathrm{z}$ found 335.1641; $\mathrm{C}_{22} \mathrm{H}_{23} \mathrm{O}_{3}\left(\mathrm{M}+\mathrm{H}^{+}\right)$requires 335.1642 .

\section{4-ethyl-2,6-bis(3-hydroxy-4-methoxybenzylidene) cyclohexanone (11)}

3-Hydroxy-4-methoxybenzaldehyde $(1.52 \mathrm{~g}, 10 \mathrm{mmol})$ and 4-ethylcyclohexanone ( $5 \mathrm{mmol}$ ) gave the title compound $\mathbf{1 1}$ as yellow crystals $(0.16 \mathrm{~g}, 8 \%), \mathrm{mp} 182-184^{\circ} \mathrm{C}$ (from $\mathrm{EtOH}) ; v_{\max }(\mathrm{KBr}) / \mathrm{cm}^{-1} 3423(\mathrm{O}-\mathrm{H}), 1647(\mathrm{C}=\mathrm{O}), 1571$ $(\mathrm{C}=\mathrm{C}), \delta_{\mathrm{H}}\left(400 \mathrm{MHz}, \mathrm{DMSO}-d_{6}\right), 0.87(3 \mathrm{H}, \mathrm{t}, J 7 \mathrm{~Hz}, \mathrm{Me})$, 1.35-1.42 (2H, m, $\left.\mathrm{CH}_{2} \mathrm{Me}\right), 1.51-1.63(1 \mathrm{H}, \mathrm{m}, \mathrm{H}-4), 2.52$ $\left(2 \mathrm{H}, \mathrm{t}, J 16 \mathrm{~Hz}, \mathrm{H}-3_{\mathrm{ax}}, 5_{\mathrm{ax}}\right), 3.01\left(2 \mathrm{H}, \mathrm{d}, J 15 \mathrm{~Hz}, \mathrm{H}-3_{\mathrm{eq}}, 5_{\mathrm{eq}}\right)$, $3.82(6 \mathrm{H}, \mathrm{s}, \mathrm{OMe}), 6.96-7.03(6 \mathrm{H}, \mathrm{m}, \mathrm{Ar}-\mathrm{H}), 7.51(2 \mathrm{H}$, $\mathrm{s},=\mathrm{CH}), 9.21(1 \mathrm{H}$, br s, OH $) ; \delta_{\mathrm{C}}\left(101 \mathrm{MHz}, \mathrm{DMSO}-d_{6}\right)$, $11.3\left(\mathrm{CH}_{3} \mathrm{CH}_{2}\right), 27.9\left(\mathrm{CH}_{3} \mathrm{CH}_{2}\right), 33.4(\mathrm{C}-3,5), 34.9(\mathrm{C}-4)$, $55.6(\mathrm{OMe}), 112.0(\mathrm{CH}), 117.1(\mathrm{CH}), 122.9(\mathrm{CH}), 128.3$ $\left(\mathrm{C}_{\text {quat }}\right), 133.1\left(\mathrm{C}_{\text {quat }}\right), 136.1(=\mathrm{CH}), 146.3(\mathrm{C}-\mathrm{O}), 148.5$ $(\mathrm{C}-\mathrm{O}), 188.6(\mathrm{C}=\mathrm{O})$; HRMS (ESI) $\mathrm{m} / \mathrm{z}$ found 395.1851; calculated for $\mathrm{C}_{24} \mathrm{H}_{27} \mathrm{O}_{5}\left(\mathrm{M}+\mathrm{H}^{+}\right) 395.1853$.

\section{4-ethyl-2,6-bis(4-hydroxy-3-methoxybenzylidene) cyclohexanone (12)}

Vanillin (1.52 g, $10 \mathrm{mmol})$ and 4-ethylcyclohexanone $(0.63 \mathrm{~g}, 5 \mathrm{mmol})$ reacted to give a crude product which was dissolved in chloroform, then applied to a plug of silica gel. Elution with $\mathrm{MeCN}$, pooling and evaporation of appropriate fractions, then recrystallization from $\mathrm{EtOH}$ gave the title compound $12(0.20 \mathrm{~g}, 10 \%)$ as a dark yellow powder mp $183-184^{\circ} \mathrm{C} ; v_{\max }(\mathrm{KBr}) / \mathrm{cm}^{-1} 3337(\mathrm{O}-\mathrm{H})$, $1647(\mathrm{C}=\mathrm{O}), 1577(\mathrm{C}=\mathrm{C}), \delta_{\mathrm{H}}\left(400 \mathrm{MHz}, \mathrm{DMSO}-d_{6}\right), 0.86$ $(3 \mathrm{H}, \mathrm{t}, J 7 \mathrm{~Hz}, \mathrm{Me}), 1.37-1.40\left(2 \mathrm{H}, \mathrm{m}, \mathrm{CH}_{2}\right), 1.51-1.64$ $(1 \mathrm{H}, \mathrm{m}, \mathrm{H}-4), 2.50-2.57(2 \mathrm{H}, \mathrm{m}$, overlapping with DMSO$\left.d_{5}, \mathrm{H}-3_{\mathrm{ax}}, 5_{\mathrm{ax}}\right), 3.03\left(2 \mathrm{H}, \mathrm{d}, J 16 \mathrm{~Hz}, \mathrm{H}-3_{\mathrm{eq}}, 5_{\mathrm{eq}}\right), 3.82(6 \mathrm{H}, \mathrm{s}$, OMe), $6.86\left(2 \mathrm{H}, \mathrm{d}, J 8 \mathrm{~Hz}, \mathrm{H}-5^{\prime}, 5^{\prime \prime}\right), 7.03(2 \mathrm{H}, \mathrm{dd}, J 8$, $\left.1.5 \mathrm{~Hz}, \mathrm{H}-6^{\prime}, 6^{\prime \prime}\right), 7.11\left(2 \mathrm{H}, \mathrm{d}, J 1.5 \mathrm{~Hz}, \mathrm{H}-2^{\prime}, 2^{\prime \prime}\right), 7.58(2 \mathrm{H}$, $\mathrm{s}, \mathrm{CH}=), 9.51(2 \mathrm{H}, \mathrm{s}, \mathrm{OH}) ; \delta_{\mathrm{C}}\left(101 \mathrm{MHz}, \mathrm{DMSO}-d_{6}\right), 11.3$ $\left(\mathrm{CH}_{3} \mathrm{CH}_{2}\right), 27.9\left(\mathrm{CH}_{3} \mathrm{CH}_{2}\right), 33.4(\mathrm{C}-3,5), 35.0(\mathrm{C}-4), 55.6$ (OMe), $114.9\left(\mathrm{C}-2^{\prime}\right), 115.6\left(\mathrm{C}-5^{\prime}\right), 124.1\left(\mathrm{C}^{\prime} 6^{\prime}\right), 126.9$ $\left(\mathrm{C}_{\text {quat }}\right), 132.6\left(\mathrm{C}_{\text {quat }}\right), 136.4(=\mathrm{CH}), 147.4(\mathrm{C}-\mathrm{O}), 147.8$ $(\mathrm{C}-\mathrm{O}), 188.5(\mathrm{C}=\mathrm{O})$; HRMS (ESI) $\mathrm{m} / \mathrm{z}$ found 395.1855; calculated for $\mathrm{C}_{24} \mathrm{H}_{27} \mathrm{O}_{5}\left(\mathrm{M}+\mathrm{H}^{+}\right) 395.1853$.

\section{2,6-bis(3-fluoro-4-hydroxybenzylidene)-4- ethylcyclohexanone (13)}

4-Ethylcyclohexanone $(0.295 \mathrm{~mL}, 2.14 \mathrm{mmol})$ and 3 fluoro-4-hydroxybenzaldehyde $(0.90 \mathrm{~g}, 6.42 \mathrm{mmol})$ after heating at $30^{\circ} \mathrm{C}$ for $15 \mathrm{~h}$ and $60^{\circ} \mathrm{C}$ for $6 \mathrm{~h}$ followed by recrystallization $(\mathrm{EtOH})$ gave the title compound $\mathbf{1 3}$ as a yellow-green powder $(0.16 \mathrm{~g}, 20 \%), \mathrm{mp} 183-185^{\circ} \mathrm{C} ; v_{\max }$ $(\mathrm{KBr}) / \mathrm{cm}^{-1} 3153(\mathrm{O}-\mathrm{H}), 1653(\mathrm{C}=\mathrm{O}), 1589(\mathrm{C}=\mathrm{C}) ; \delta_{\mathrm{H}}$ $\left(400 \mathrm{MHz}, \mathrm{DMSO}-d_{6}\right), 0.86\left(3 \mathrm{H}, \mathrm{t}, J 7 \mathrm{~Hz}, \mathrm{CH}_{3} \mathrm{CH}_{2}\right), 1.39$ ( $2 \mathrm{H}$, quintet, $\left.J 7 \mathrm{~Hz}, \mathrm{CH}_{3} \mathrm{CH}_{2}\right), 1.57-1.62(1 \mathrm{H}, \mathrm{m}, \mathrm{H}-4)$, 2.45-2.60 $\left(2 \mathrm{H}, \mathrm{m}\right.$, overlapping with DMSO- $d_{5}, \mathrm{H}-3_{\mathrm{ax}}$, $\left.5_{\text {ax }}\right), 2.98\left(2 \mathrm{H}, \mathrm{dd}, J 16,3 \mathrm{~Hz}, \mathrm{H}-3_{\mathrm{eq}}, 5_{\mathrm{eq}}\right), 7.03(2 \mathrm{H}, \mathrm{t}, J$ $\left.9 \mathrm{~Hz}, \mathrm{H}-5^{\prime}, 5^{\prime \prime}\right), 7.21\left(2 \mathrm{H}, \mathrm{dd}, J 9,2 \mathrm{~Hz}, \mathrm{H}-6^{\prime}, 6^{\prime \prime}\right), 7.38$ $\left(2 \mathrm{H}, \mathrm{dd}, J 13,2 \mathrm{~Hz}, \mathrm{H}-2^{\prime}, 2^{\prime \prime}\right), 7.53(2 \mathrm{H}, \mathrm{s}, \mathrm{CH}=\mathrm{C}) ; \delta_{\mathrm{C}}$ $\left(101 \mathrm{MHz}\right.$, DMSO- $\left.d_{6}\right), 11.3\left(\mathrm{CH}_{3} \mathrm{CH}_{2}\right), 27.7\left(\mathrm{CH}_{3} \mathrm{CH}_{2}\right)$, 33.1 (C-3,5), 34.8 (C-4), 117.7 (d, J $\left.3 \mathrm{~Hz}, \mathrm{C}-5^{\prime}, 5^{\prime \prime}\right), 118.0$ $\left(\mathrm{d}, J 18 \mathrm{~Hz}, \mathrm{C}-2^{\prime}, 2^{\prime \prime}\right), 127.0\left(\mathrm{C}-1^{\prime}, 1^{\prime \prime}\right), 127.7\left(\mathrm{C}-6^{\prime}, 6^{\prime \prime}\right)$, $133.6\left(=\mathrm{C}_{\text {quat }}\right), 135.1(=\mathrm{CH}), 145.9\left(\mathrm{~d}, J 12 \mathrm{~Hz}, \mathrm{C}-4^{\prime}, 4^{\prime \prime}\right)$, $150.6(\mathrm{~d}, J 241 \mathrm{~Hz}, \mathrm{C}-\mathrm{F}), 188.5(\mathrm{C}=\mathrm{O}) ; \delta_{\mathrm{F}}(376 \mathrm{MHz}$, DMSO- $d_{6}$ ), -132.3 ; HRMS (ESI) $\mathrm{m} / \mathrm{z}$ found 371.1451 ; calculated for $\mathrm{C}_{22} \mathrm{H}_{21} \mathrm{~F}_{2} \mathrm{O}_{3}\left(\mathrm{M}+\mathrm{H}^{+}\right) 371.1453$.

\section{4-tert-butyl-2,6-bis(4-hydroxy-3-} methoxybenzylidene)cyclohexanone (15)

Vanillin $(1.52 \mathrm{~g}, 10 \mathrm{mmol})$ and 4-tert-butylcyclohexanone $(0.77 \mathrm{~g}, 5 \mathrm{mmol})$ gave the title compound $15(0.61 \mathrm{~g}$, $30 \%$ ) as yellow crystals, mp $194-196{ }^{\circ} \mathrm{C}$ (from $\mathrm{EtOH}$ ); $v_{\max }(\mathrm{KBr}) / \mathrm{cm}^{-1} 3337(\mathrm{O}-\mathrm{H}), 1647(\mathrm{C}=\mathrm{O}), 1589(\mathrm{C}=\mathrm{C})$, $\delta_{\mathrm{H}}\left(400 \mathrm{MHz}, \mathrm{DMSO}-d_{6}\right) 0.94\left(9 \mathrm{H}, \mathrm{s}, \mathrm{Me}_{3} \mathrm{C}\right), 1.32-1.42$ $(1 \mathrm{H}, \mathrm{m}, \mathrm{H}-4), 2.42-2.52(2 \mathrm{H}, \mathrm{m}$, overlapping with DMSO- $d_{5}$ signal, $\left.\mathrm{H}-3_{\mathrm{ax}}, 5_{\mathrm{ax}}\right), 3.08\left(2 \mathrm{H}, \mathrm{d}, J 14 \mathrm{~Hz}, \mathrm{H}-3_{\text {eq }}\right.$, $\left.5_{\mathrm{eq}}\right), 3.81(6 \mathrm{H}, \mathrm{s}, \mathrm{OMe}), 6.88\left(2 \mathrm{H}, \mathrm{d}, J 8 \mathrm{~Hz}, \mathrm{H}-5^{\prime}, 5^{\prime \prime}\right)$, $7.04\left(2 \mathrm{H}, \mathrm{dd}, J 8,2 \mathrm{~Hz}, \mathrm{H}-6^{\prime}, 6^{\prime \prime}\right), 7.12(2 \mathrm{H}, \mathrm{d}, J 2 \mathrm{~Hz}, \mathrm{H}-$ $\left.2^{\prime}, 2^{\prime \prime}\right), 7.56(2 \mathrm{H}, \mathrm{d}, J 2 \mathrm{~Hz},=\mathrm{CH}), 9.52(2 \mathrm{H}, \mathrm{br} \mathrm{s}, \mathrm{OH}) ; \delta_{\mathrm{C}}$ (101 MHz, DMSO- $\left.d_{6}\right), 27.1\left(\mathrm{Me}_{3} \mathrm{C}\right), 29.1(\mathrm{C}-3,5), 32.1$ $\left(\mathrm{Me}_{3} \mathrm{C}\right), 43.8(\mathrm{C}-4), 55.6(\mathrm{MeO}), 114.7\left(\mathrm{C}-2^{\prime}, 2^{\prime \prime}\right), 115.6$ $\left(\mathrm{C}-5^{\prime}, 5^{\prime \prime}\right), 124.2\left(\mathrm{C}-6^{\prime}, 6^{\prime \prime}\right), 126.9\left(\mathrm{C}_{\text {quat }}\right), 133.3\left(\mathrm{C}_{\text {quat }}\right)$, $136.1(=\mathrm{CH}), 147.4(\mathrm{C}-\mathrm{O}), 147.8(\mathrm{C}-\mathrm{O}), 188.5(\mathrm{C}=\mathrm{O})$; HRMS (ESI) $\mathrm{m} / \mathrm{z}$ found 423.2163; calculated for $\mathrm{C}_{26} \mathrm{H}_{31} \mathrm{O}_{5}\left(\mathrm{M}+\mathrm{H}^{+}\right) 423.2166$.

\section{2,5-bis(3-hydroxy-4-methoxybenzylidene) tetrahydro-4H-pyran-4-one (17)}

3-Hydroxy-4-methoxybenzaldehyde $\quad(1.52 \mathrm{~g}, \quad 10 \mathrm{mmol})$, tetrahydro- $4 H$-pyran-4-one $(0.51 \mathrm{~g}, 5.09 \mathrm{mmol}), \mathrm{MeOH}$ $(5 \mathrm{~mL})$ concentrated $\mathrm{HCl}(1 \mathrm{~mL})$ were heated together at $30{ }^{\circ} \mathrm{C}$ for $15 \mathrm{~h} .50 \%$ Aqueous $\mathrm{AcOH}(20 \mathrm{~mL})$ was added and the crude product was filtered off, then recrystallized from $\mathrm{EtOH}$ to give the title compound $\mathbf{1 7}$ as yellow crystals $(0.36 \mathrm{~g}, 20 \%), \mathrm{mp} 272-273{ }^{\circ} \mathrm{C} ; v_{\max }(\mathrm{KBr}) / \mathrm{cm}^{-1} 3294$ $(\mathrm{O}-\mathrm{H}), 1663(\mathrm{C}=\mathrm{O}), 1597(\mathrm{C}=\mathrm{C}) ; \delta_{\mathrm{H}}(400 \mathrm{MHz}, \mathrm{DMSO}-$ $\left.d_{6}\right) 3.83(6 \mathrm{H}, \mathrm{s}, \mathrm{OMe}), 4.88\left(4 \mathrm{H}, \mathrm{s}, \mathrm{OCH}_{2}\right), 6.87-6.91(4 \mathrm{H}$, m), $7.01(2 \mathrm{H}, \mathrm{d}, J 8 \mathrm{~Hz}), 7.53(2 \mathrm{H}, \mathrm{s},=\mathrm{CH}), 9.28(2 \mathrm{H}, \mathrm{br} \mathrm{s}$, $\mathrm{OH}) ; \delta_{\mathrm{C}}\left(101 \mathrm{MHz}, \mathrm{DMSO}-d_{6}\right) 55.6(\mathrm{OMe}), 67.8\left(\mathrm{OCH}_{2}\right)$, $112.1(\mathrm{CH}), 117.3(\mathrm{CH}), 123.3(\mathrm{CH}), 127.1\left(\mathrm{C}_{\text {quat }}\right), 131.5$ 
$\left(\mathrm{C}_{\text {quat }}\right), 135.0(=\mathrm{CH}), 146.5(\mathrm{C}-\mathrm{O}), 149.2(\mathrm{C}-\mathrm{O}), 184.4$ $(\mathrm{C}=\mathrm{O}$ ); HRMS (ESI) $\mathrm{m} / \mathrm{z}$ found 369.1332; calculated for $\mathrm{C}_{21} \mathrm{H}_{21} \mathrm{O}_{6}\left(\mathrm{M}+\mathrm{H}^{+}\right) 399.1333$.

\section{2,5-bis(3-fluoro-4-hydroxybenzylidene) tetrahydropyran-4-one (18)}

Tetrahydropyran-4-one $\quad(0.198 \mathrm{~mL}, \quad 2.14 \mathrm{mmol}), \quad 3-$ fluoro-4-hydroxybenzaldehyde $(0.60 \mathrm{~g}, 4.28 \mathrm{mmol})$ and methanol $(2.14 \mathrm{~mL})$, after heating at $60{ }^{\circ} \mathrm{C}$ for $2 \mathrm{~d}$, gave a crude product that was subjected to flash chromatography $\left(\mathrm{CH}_{2} \mathrm{Cl}_{2}\right.$-EtOAc, 9:1) followed by recrystallization $(\mathrm{EtOH})$ to give the title compound $\mathbf{1 8}$ as a yellow powder $(0.158 \mathrm{~g}, 21 \%) ; \mathrm{mp} 234-236{ }^{\circ} \mathrm{C} ; v_{\max }(\mathrm{KBr}) / \mathrm{cm}^{-1}$ $3237(\mathrm{O}-\mathrm{H}), 1666(\mathrm{C}=\mathrm{O}), 1605(\mathrm{C}=\mathrm{C}) ; \delta_{\mathrm{H}}(400 \mathrm{MHz}$, DMSO- $\left.d_{6}\right), 4.86\left(4 \mathrm{H}, \mathrm{s}, \mathrm{OCH}_{2}\right), 7.03\left(2 \mathrm{H}, \mathrm{t}, J 9 \mathrm{~Hz}, \mathrm{H}-5^{\prime}\right.$, $\left.5^{\prime \prime}\right), 7.10\left(2 \mathrm{H}, \mathrm{dd}, J 9,2 \mathrm{~Hz}, \mathrm{H}-6^{\prime}, 6^{\prime \prime}\right), 7.26(2 \mathrm{H}, \mathrm{dd}, J 13$, $\left.2 \mathrm{~Hz}, \mathrm{H}-2^{\prime}, 2^{\prime \prime}\right), 7.55(2 \mathrm{H}, \mathrm{s}, \mathrm{CH}=) ; \delta_{\mathrm{C}}(101 \mathrm{MHz}$, DMSO- $\left.d_{6}\right) 67.6\left(\mathrm{OCH}_{2}\right), 117.9\left(\mathrm{~d}, J 3 \mathrm{~Hz}, \mathrm{C}-5^{\prime}, 5^{\prime \prime}\right)$, $118.4\left(\mathrm{~d}, J 18 \mathrm{~Hz}, \mathrm{C}-2^{\prime}, 2^{\prime \prime}\right), 125.8$ (d, $\left.J 6 \mathrm{~Hz}, \mathrm{C}-1^{\prime}, 1^{\prime \prime}\right)$, $128.0\left(\mathrm{~d}, J 2 \mathrm{~Hz}, \mathrm{C}-6^{\prime}, 6^{\prime \prime}\right), 131.7\left(=\mathrm{C}_{\text {quat }}\right), 134.0(=\mathrm{CH})$, 146.6 (d, $J 13 \mathrm{~Hz}, \mathrm{C}-\mathrm{O}), 150.7$ (d, $J 242 \mathrm{~Hz}, \mathrm{CF}), 184.4$ $(\mathrm{C}=\mathrm{O}) ; \delta_{\mathrm{F}}\left(376 \mathrm{MHz}\right.$, DMSO- $\left.d_{6}\right),-131.9$; HRMS (ESI-) $\mathrm{m} / \mathrm{z}$ found 343.0789 ; calculated for $\mathrm{C}_{19} \mathrm{H}_{13} \mathrm{~F}_{2} \mathrm{O}_{4}\left(\mathrm{M}-\mathrm{H}^{+}\right)$ 343.0787 .

\section{Method of cell culture and viability assay}

Human ovarian carcinoma cells sensitive to cisplatin, A2780 and resistant to cisplatin, A2780-CP70 were grown and maintained in RPMI 1640 medium supplemented with $10 \%$ fetal bovine serum at $37{ }^{\circ} \mathrm{C}, 5 \% \mathrm{CO}_{2}$. The cells were plated in 96-well culture plates at a density of $1 \times 10^{4}$ cells/ well and allowed to adhere at $37^{\circ} \mathrm{C}$ for $24 \mathrm{~h}$. Then the next day the cells were treated with various doses of compounds ranging from $0.1 \mathrm{nM}$ to $10 \mu \mathrm{M}$ or vehicle. Cell viability assay, MTT, was performed following incubation time of $96 \mathrm{~h}$. In such experiments the supernatant was removed and MTT (3-(4,5-dimethylthiazol-2-yl)-2,5diphenyltetrazolium bromide) was added for $4 \mathrm{~h}$. The ability of cells to form formazan crystals by active mitochondrial respiration was determined by using a microplate reader after dissolving the crystals in DMSO. Cytotoxicity was expressed as a relative percentage of the absorbance measured at $540 \mathrm{~nm}$ in the control and drugtreated cells. MTT assay (Mosmann 1983) measures the conversion of the soluble tetrazolium salt (MTT) to an insoluble purple formazan by succinate dehydrogenase in the mitochondria of living cells. $\mathrm{IC}_{50}$ values are reported as mean \pm s.e. mean $(n=2-4$ experiments). Carboplatin (20) was used as a reference chemotherapeutic drug.

\section{Results and discussion}

The aldol condensation reaction between aromatic aldehydes and aliphatic ketones in appropriate stoichiometric proportions gives $\alpha, \alpha^{\prime}$-bis(arylidene)ketones (Scheme 1). Use of hydroxybenzaldehyde starting materials readily allows the construction of symmetrical products that resemble curcumin in the sense that two phenol or catechol rings are connected by a linking group containing an unsaturated ketone. Some substances of this general type are known to have activity against various cancer cell lines. These include HeLa human cervix cancer (Costi et al. 2004), leukemia (Tan et al. 2014), and HEKT293T human embryonic kidney cancer (Leow et al. 2014).

There is literature precedent for conducting aldol condensation reactions under either acidic or basic conditions. However, base would deprotonate phenolic hydroxyl groups that are present in the aldehyde substrates, probably necessitating the protection of the hydroxyl groups (Artico et al. 1998). We therefore decided to perform the reactions directly on the hydroxybenzaldehydes in the presence of hydrochloric acid (general approach of Sardjiman et al. 1997). Examples were selected to investigate the effect of including methoxyl or fluorine atom substituents in addition to the phenolic hydroxyl groups.

Synthetic ketones 2-19 were readily isolated as crystalline solids that had NMR, IR, and high resolution mass spectra consistent with the expected structures. The cyclopentanone derivative $\mathbf{2}$ did not show significant inhibition of the growth of either ovarian cell line at concentrations up to $10 \mu \mathrm{M}$ (Table 1); cyclopentanone 4 was also ineffective against cisplatin-resistant cells. However, all of the cyclohexanones 5-15 and heterocyclic analogs 16-19, showed inhibition with $\mathrm{IC}_{50}<10 \mu \mathrm{M}$, including against cisplatin-resistant cells. This contrasts with the results obtained using carboplatin 20, which is used to treat ovarian cancer clinically, but is comparatively ineffective against cisplatin-resistant cells. It is notable that the cyclohexanone and the 4ethylcyclohexanone derivatives $\mathbf{5}$ and $\mathbf{1 1}$ are much more active than cyclopentanone $\mathbf{2}$, which has identically substituted aromatic rings. Alkyl substitution of the 4position of the cyclohexanone ring is well tolerated, even with the bulky tert-butyl group in analogs $\mathbf{1 4}$ and $\mathbf{1 5}$. It is also evident that the pyranones 16-18 and the thiopyranone 19 have high levels of activity against ovarian cancer cells. These results should be compared with previous studies in which thiopyranone derivatives were found to be highly active against both NB4 acute promyelocytic leukemic cells and NB4-R1 retinoic acid resistant cells (Tan et al. 2014), and a piperidin-4-one 
Table 1 Biological activity ( $\mathrm{IC}_{50}$ in MTT assay) of synthetic curcumin analogs 2-19 against A2780 ovarian cancer cells and A2780-CP70 cisplatin-resistant ovarian cancer cells<smiles>[R]c1ccc(/C=C2\CC/C(=C\c3ccc([R2])c([R])c3)C2=O)cc1[R]</smiles>

2 - 4<smiles>[X]CC(=Cc1ccc([R6])c([R])c1)C=C1C=CC([R])=C([R])C1</smiles>

$5-19$<smiles>CCCC1(C(=O)O[PH3](N)(N)N)OC1=O</smiles>

20

\begin{tabular}{llllll}
\hline Compound & $\mathrm{X}$ & $R^{1}$ & $R^{2}$ & $\begin{array}{l}\mathrm{IC}_{50} \\
(\mu \mathrm{M}) \mathrm{A} 2780\end{array}$ & $\begin{array}{l}\mathrm{IC}_{50}(\mu \mathrm{M}) \\
\mathrm{A} 2780-\mathrm{CP} 70\end{array}$ \\
\hline 2 & - & $\mathrm{OH}$ & $\mathrm{OMe}$ & $>10$ & $>10$ \\
3 & - & $\mathrm{OMe}$ & $\mathrm{OH}$ & $0.043 \pm 0.01$ & $0.458 \pm 0.1$ \\
4 & - & $\mathrm{F}$ & $\mathrm{OH}$ & $0.749 \pm 0.25$ & $>10$ \\
5 & $\mathrm{CH}_{2}$ & $\mathrm{OH}$ & $\mathrm{OMe}$ & $0.595 \pm 0.09$ & $0.71 \pm 0.1$ \\
6 & $\mathrm{CH}_{2}$ & $\mathrm{OMe}$ & $\mathrm{OH}$ & $0.28 \pm 0.08$ & $2.21 \pm 0.05$ \\
7 & $\mathrm{CH}_{2}$ & $\mathrm{~F}$ & $\mathrm{OH}$ & $0.99 \pm 0.5$ & $3.8 \pm 0.09$ \\
8 & $\mathrm{CHMe}$ & $\mathrm{OMe}$ & $\mathrm{OH}$ & $6.59 \pm 0.6$ & $1.69 \pm 0.71$ \\
9 & $\mathrm{CHMe}$ & $\mathrm{F}$ & $\mathrm{OH}$ & $8.19 \pm 0.29$ & $3.24 \pm 0.27$ \\
10 & $\mathrm{CHEt}$ & $\mathrm{H}$ & $\mathrm{OH}$ & $5.32 \pm 1.1$ & $3.065 \pm 0.62$ \\
11 & $\mathrm{CHEt}$ & $\mathrm{OH}$ & $\mathrm{OMe}$ & $0.15 \pm 0.09$ & $1.25 \pm 0.09$ \\
12 & $\mathrm{CHEt}$ & $\mathrm{OMe}$ & $\mathrm{OH}$ & $0.36 \pm 0.08$ & $1.82 \pm 0.44$ \\
13 & $\mathrm{CHEt}$ & $\mathrm{F}$ & $\mathrm{OH}$ & $2.04 \pm 0.35$ & $3.38 \pm 0.3$ \\
14 & $\mathrm{CH}{ }^{t} \mathrm{Bu}$ & $\mathrm{OH}$ & $\mathrm{OH}$ & $2.86 \pm 1.74$ & $3.48 \pm 0.8$ \\
15 & $\mathrm{CH}{ }^{t} \mathrm{Bu}$ & $\mathrm{OMe}$ & $\mathrm{OH}$ & $3.95 \pm 1.5$ & $2.34 \pm 0.3$ \\
16 & $\mathrm{O}$ & $\mathrm{OH}$ & $\mathrm{OH}$ & $0.178 \pm 0.04$ & $1.29 \pm 0.98$ \\
17 & $\mathrm{O}$ & $\mathrm{OH}$ & $\mathrm{OMe}$ & $0.26 \pm 0.03$ & $0.367 \pm 0.01$ \\
18 & $\mathrm{O}$ & $\mathrm{F}$ & $\mathrm{OH}$ & $1.65 \pm 0.9$ & $2.67 \pm 0.4$ \\
19 & $\mathrm{~S}$ & $\mathrm{OMe}$ & $\mathrm{OH}$ & $0.129 \pm 0.07$ & $0.153 \pm 0.064$ \\
20 (carboplatin) & & & $2.57 \pm 0.35$ & $44.9 \pm 3.5$ \\
\hline
\end{tabular}

$\mathrm{IC}_{50}$ data for carboplatin $\mathbf{2 0}$ are shown for comparison

derivative was active against cisplatin-resistant ovarian cancer cells (Selvendiran et al. 2007).

With regard to the substituents on the aryl groups, we observe that replacing $R^{1}=\mathrm{OMe}$ in 8 by $R^{1}=\mathrm{F}$ in 9 did not improve activity, neither did replacement of $R^{1}=\mathrm{OH}$ in $\mathbf{1 6}$ to give 18, whereas substituting $R^{1}=\mathrm{H}$ in compound $\mathbf{1 0}$ by $R^{1}=\mathrm{F}$ to give $\mathbf{1 3}$ was of limited benefit.

It is known that the enones such as these are able to react as electrophiles, undergoing addition of soft nucleophiles such as thiols to the $\beta$-carbon of the $\mathrm{C}=\mathrm{C}$ bond. It has previously been shown that such reactions are responsible for the induction of phase 2 enzymes such as quinone reductase (Dinkova-Kostova et al. 2001). To compare the electrophilicity of representative compounds from the present study, we ran a series of ${ }^{1} \mathrm{H}$ NMR spectra on samples in which individual samples of cyclopentanone $\mathbf{2}$, cyclohexanone $\mathbf{5}$, and tetrahydropyranone 17 were mixed with a model thiol (3mercaptopropionic acid) in a 1:20 molar ratio. After a few hours it was observed that the signal intensities due to the $\mathrm{C}=\mathrm{CH}$ protons diminished (Fig. 1). These signals are singlets which occur downfield of the aromatic signals, with chemical shifts between 7.3 and $7.6 \mathrm{ppm}$. The appearance of new signals between 6.6-7.0 and 3.7-4.0 ppm was consistent with Michael addition having occurred in each case, with the cyclopentanone 2 clearly being less reactive than the six-membered ring analogs. Analysis of data obtained at different times indicated that the pseudo first order rate constant for consumption of cyclohexanone $\mathbf{5}$ was 5.1 times greater than that of the analogous cyclopentanone $\mathbf{2}$, whereas that for the tetrahydropyranone $\mathbf{1 7}$ was 8.0 times greater than that of $\mathbf{2}$. Thus, for these three compounds, the trend in electrophilicity correlates with cytotoxicity. 
Fig. $1400 \mathrm{MHz}{ }^{1} \mathrm{H}$ NMR spectra of mixtures of individual ketones $\left(1.00 \times 10^{-5} \mathrm{~mol}\right)$ with 3-mercaptopropanoic acid $\left(2.00 \times 10^{-4} \mathrm{~mol}\right)$ in DMSO- $d_{6}$ $(0.60 \mathrm{~mL})$ at $21^{\circ} \mathrm{C}$. Spectra shown were recorded $0.5 \mathrm{~h}$ (left) and $4.0 \mathrm{~h}$ (right) after sample preparation. Ketones used were cyclopentanone 2 (top spectra), cyclohexanone $\mathbf{5}$ (middle spectra), and tetrahydropyranone 17 (bottom spectra)
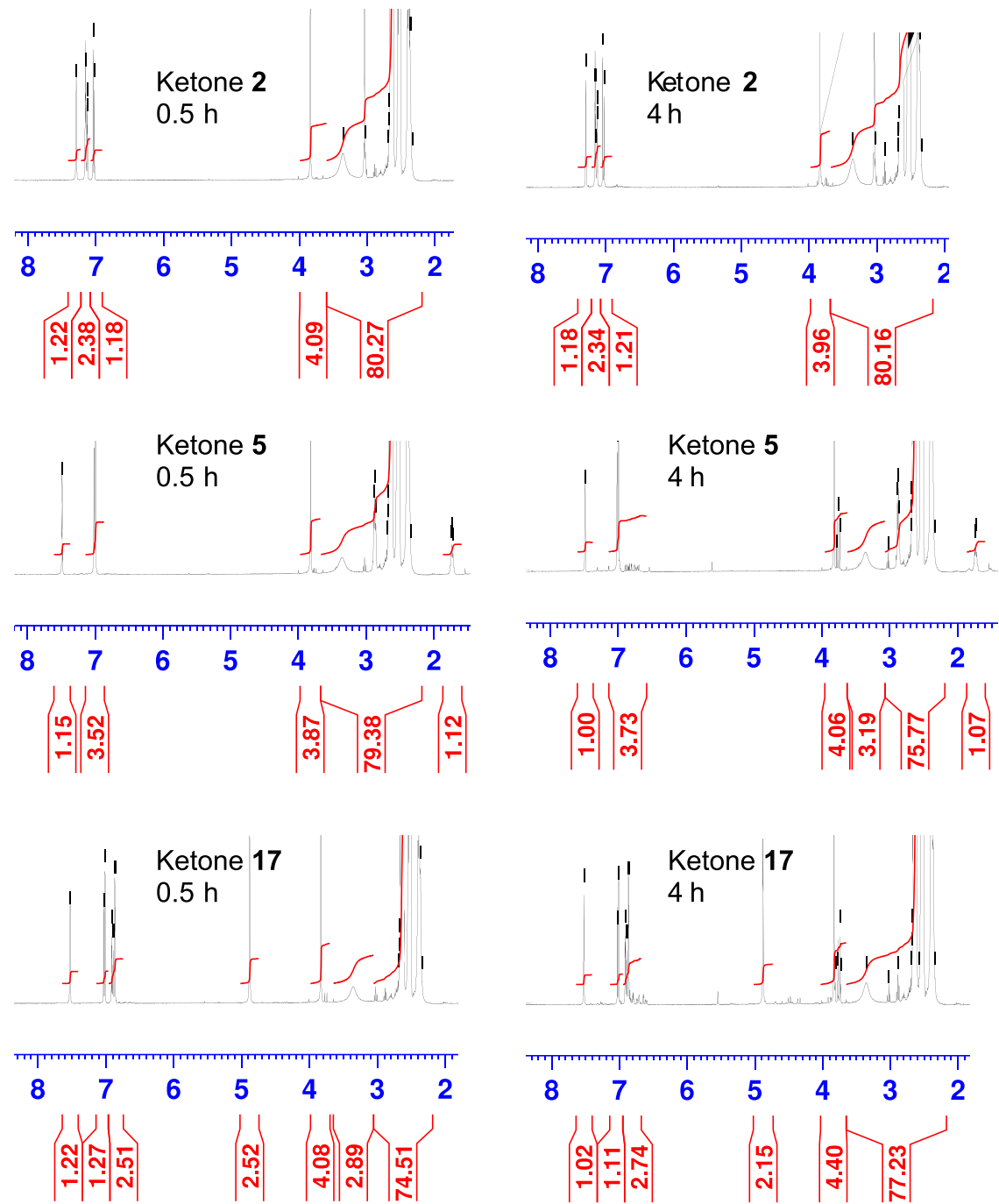

The fact that cisplatin-resistant cells were sensitive to the cytotoxicity induced by these compounds also indicates that the mechanism(s) of action of these compounds are likely to be independent of nucleus events associated with the DNA repair cascade as platinum compounds primarily affect the DNA within the nucleus. Organic compounds that target DNA, such as nitrogen mustards and anthraquinones, show strong cross-resistance with cisplatin (Hamaguchi et al. 1993). However, other non DNA-related damage can also lead to apoptosis or cell death. For example, it has been shown that changes in the mitochondrial function following the release of cytochrome $c$ into the cytosol resulted in caspase activation, which in turn leads to apoptosis (Liu et al. 1996). This indicated that apoptosis can also be initiated in the cell cytosol and not just the nucleus (Cory and Adams 2002). Considering that many patients do develop resistance to platinum drugs would highlight the importance of developing analogues that at least initially activate the apoptotic pathway in the cytoplasm of the cells, which bypasses the resistance pathway. Further experiments are required to substantiate the mechanism of action of these compounds.

\section{Conclusions}

Most of the synthetic curcumin analogues that were studied showed significant inhibition of ovarian cancer cell growth, including inhibition of cisplatin-resistant cells. The ability of the enone $\mathrm{C}=\mathrm{C}$ groups in these analogues react electrophilically with thiol groups of biological molecules is likely to be relevant to their activity. The derivatives of the heterocyclic ketones stand out as being particularly active and worthy of further investigation. 
Acknowledgements We are grateful to the EPSRC NMSF at Swansea University for mass spectrometry.

\section{Compliance with ethical standards}

Conflict of interest The authors declare that they have no conflict of interest.

Publisher's note Springer Nature remains neutral with regard to jurisdictional claims in published maps and institutional affiliations.

Open Access This article is licensed under a Creative Commons Attribution 4.0 International License, which permits use, sharing, adaptation, distribution and reproduction in any medium or format, as long as you give appropriate credit to the original author(s) and the source, provide a link to the Creative Commons license, and indicate if changes were made. The images or other third party material in this article are included in the article's Creative Commons license, unless indicated otherwise in a credit line to the material. If material is not included in the article's Creative Commons license and your intended use is not permitted by statutory regulation or exceeds the permitted use, you will need to obtain permission directly from the copyright holder. To view a copy of this license, visit http://creativecommons. org/licenses/by/4.0/.

\section{References}

Adams BK, Ferstl EM, Davis MC, Herold M, Kurtkayer S, Camalier RF, Hollingshead MG, Kaur G, Sausville EA, Rickles FR, Snyder JP, Liotta DC, Shoji M (2004) Synthesis and biological evaluation of novel curcumin analogs as anti-cancer and antiangiogenesis agents. Bioorg Med Chem 12:3871-3883

Artico M, Di Santo R, Costi R, Novellino E, Greco G, Massa S, Tramontano E, Marongiu ME, De Montis A, La Colla P (1998) Geometrically and conformationally restrained cinnamoylcompounds as inhibitors of HIV-1 integrase: synthesis, biological evaluation and molecular modeling. J Med Chem 41:3948-3960

Bairam R, Muppavarapu S, Sreekanth S (2017) Synthesis, characterization, biological evaluation and docking of some novel substituted 1,3-thiazine derivatives. Int J Pharm Pharm Sci 9:233-242

Cory S, Adams JM (2002) The BCL family: regulators of the cellular life-or-death switch. Nat Rev Cancer 2:647-656

Costi R, Di Santo R, Artico M, Massa S, Ragno R, Loddo R, La Colla M, Tramontano E, La Colla P, Pani A (2004) 2,6-Bis(3,4,5-trihydroxybenzylydene) derivatives of cyclohexanone: novel potent HIV-1 integrase inhibitors that prevent HIV-1 multiplication in cell-based assays. Bioorg Med Chem 12:199-215
Dinkova-Kostova AT, Massiah MA, Bozak RE, Hicks RJ, Talalay P (2001) Potency of Michael reaction acceptors as inducers of enzymes that protect against carcinogenesis depends on their reactivity with suflhydryl groups. PNAS 98:3404-3409

Galluzzi G, Vitale I, Michels J, Brenner C, Szabadkai G, Harel-Bellan A, Castedo M, Kroemer G (2014) Systems biology of cisplatin resistance: past, present and future. Cell Death Dis 5:e1257

Hamaguchi K, Godwin AK, Yakushiji M, O'Dwyer PJ, Ozols RF, Hamilton TC (1993) Cross-resistance to diverse drugs is associated with primary cisplatin resistance in ovarian cancer cell lines. Cancer Res 53:5225-5232

Inayama S, Mamoto K, Shibata T, Hirose T (1976) Structure and antitumor activity relation of 2-arylidene-4-cyclopentene-1,3diones and 2-arylideneindan-1,3-diones. J Med Chem 19: 433-436

Leow P-C, Bahety P, Boon CP, Lee CY, Tan KL, Yang T, Ee P-LR (2014) Functionalized curcumin analogs as potent modulators of the Wnt/ $\beta$-catenin signaling pathway. Eur J Med Chem 71:67-80

Liu X, Kim CN, Yang J, Jemmerson R, Wang X (1996) Induction of apoptotic program in cell-free extracts: requirement for $\mathrm{dATP}$ and cytochrome C. Cell 86:147-157

Mosmann T (1983) Rapid colorimetric assay for cellular growth and survival: application to proliferation and cytotoxicity assays. J Immunol Methods 65:55-63

Sardjiman SS, Reksohadiprodjo MS, Hakim L, van der Goot H, Timmerman H (1997) 1,5-Diphenyl-1,4-pentadiene-3-ones and cyclic analogues as antioxidative agents. Synthesis and structureactivity relationship. Eur J Med Chem 32:625-630

Selvendiran K, Tong L, Vishwanath S, Bratasz A, Trigg NJ, Kutala VK, Hideg K, Kuppusamy P (2007) EF24 induces $\mathrm{G}_{2} / \mathrm{M}$ arrest and apoptosis in cisplatin-resistant human ovarian cancer cells by increasing PTEN expression. J Biol Chem 282: 28609-28618

Tan K-L, Ali A, Du Y, Fu H, Jin H-X, Chin T-M, Khan M, Go M-L (2014) Synthesis and evaluation of bisbenzylidenedioxotetrahydrothiopyranones as activators of endoplasmic reticulum (ER) stress signaling pathways and apoptotic cell death in acute promyloctic leukemic cells. J Med Chem 57:5904-5918

Weir NM, Selvendiran K, Kutala VK, Tong L, Vishwanath S, Rajaram M, Tridandapani S, Anant S, Kuppusamy P (2007) Curcumin induces $\mathrm{G}_{2} / \mathrm{M}$ arrest and apoptosis in cisplatin-resistant human ovarian cancer cells by modulating Akt and p38 MAPK. Cancer Biol Ther 6:178-184

World Health Organization, 2019 Estimated number of deaths in 2018, worldwide, females, all ages. www.globocan.iarc.fr. Accessed 12 June 2019

Youssef KM, El-Sherbeny MA, El-Shafie FS, Farag HA, Al-Deeb OA, Awadalla SAA (2004) Synthesis of curcumin analogues as potential antioxidant, cancer chemopreventive agents. Arch Pharm Pharm Med Chem 337:42-54 